\title{
A path integral derivation of $\chi_{y}$-genus
}

\author{
Guowu Meng \\ Department of Mathematics, Hong Kong University of Science and Technology, \\ Clear Water Bay, Kowloon, Hong Kong \\ E-mail: mameng@ust.hk
}

Received 28 August 2002

Published 15 January 2003

Online at stacks.iop.org/JPhysA/36/1083

\begin{abstract}
The formula for the Hirzebruch $\chi_{y}$-genus of complex manifolds is a consequence of the Hirzebruch-Riemann-Roch formula. The classical index formulae for Todd genus, Euler number and signature correspond to the case when the complex variable $y=0,-1$ and 1 respectively. Here we give a direct derivation of this nice formula based on supersymmetric quantum mechanics.
\end{abstract}

PACS numbers: $12.60 . J v, 02.40 . \mathrm{Ma}$

Mathematics Subject Classification: Primary: 19K56, Secondary: 81 S40

\section{Introduction}

It has been known for about twenty years that the Atiyah-Singer formula for the index of Dirac operator can be directly derived from supersymmetric (SUSY) quantum mechanics $[2,3]$. Indeed, the well-known index formulae for Euler number, signature, Todd genus and $\hat{A}$-genus can each be derived directly from a simple SUSY quantum mechanic model.

In this paper, we would like to point out that the formula for the Hirzebruch $\chi_{y}$-genus on Kähler manifolds can also be derived directly from a simple SUSY quantum mechanic model.

Let $M$ be a complex $d$-dimensional Kähler manifold, $b_{i, j}$ the $(i, j)$-Hodge number of $M$, then the Hirzebruch $\chi_{y}$-genus is defined as

$$
\chi_{y}(M)=\sum_{i, j}(-1)^{i} y^{j} b_{i, j}
$$

Let $\Omega=\left[\Omega_{\bar{k} l}\right]_{1 \leqslant k, l \leqslant d}$ be the curvature 2 -form of the Kähler metric, $-\mathrm{i} \Omega_{n}(1 \leqslant n \leqslant d)$ the eigenvalue of anti-Hermitian matrix $\Omega, y$ a complex variable, $\sinh _{y}(x):=\frac{\mathrm{e}^{x}+y \mathrm{e}^{-x}}{2}$. Then, the formula for the Hirzebruch $\chi_{y}$-genus is

$$
\chi_{y}(M)=\left.\int_{M} \prod_{n} \frac{\Omega_{n} / 2 \pi}{\sinh \left(\Omega_{n} / 4 \pi\right)} \sinh _{y}\left(\Omega_{n} / 4 \pi\right)\right|_{\text {top form }} .
$$

(Note that the integrand is a symmetric polynomial in $\Omega_{1}, \ldots, \Omega_{d}$, hence it is a polynomial in traces of products of $\Omega$, i.e. a polynomial in Chern classes. Note also that since both sides of 
(2) are polynomials in $y$, we just need to derive (2) for $y$ being a complex number with unit norm.)

\section{SUSY quantum mechanics}

To begin with, following [5] (but with dimension reduced from $1+1$ to $0+1$ ), we recall the standard SUSY quantum mechanics model on a Kähler manifold $M$ with Kähler metric $g$. It governs maps $\Phi:[0, \beta] \rightarrow X$, with $[0, \beta]$ being a close interval. If we pick local coordinates $t$ on $[0, \beta]$ and $\phi^{I}$ on $M$, then $\Phi$ can be described locally via functions $\phi^{I}(t)$. The local complex coordinates on $M$ are denoted by $\phi^{i}$, and the complex conjugates are denoted by $\phi^{\bar{i}}=\overline{\phi^{i}}$. Let $T^{\prime} M$ be the holomorphic tangent bundle of $M$ and $T^{\prime \prime} M$ the antiholomorphic tangent bundle of $M$. The Fermi fields of the model are $\psi_{+}^{i}, \psi_{-}^{i}$, sections of $T M$, and $\psi_{+}^{\bar{i}}, \psi_{-}^{\bar{i}}$ sections of $T^{\prime \prime} M$. The Lagrangian is

$$
I=\int_{0}^{\beta} \mathrm{d} t\left(g_{\bar{i} j} \dot{\phi}^{\bar{i}} \dot{\phi}^{j}+\mathrm{i} g_{\bar{i} j} \psi_{-}^{\bar{i}} \nabla_{t} \psi_{-}^{j}+\mathrm{i} g_{\bar{i} j} \psi_{+}^{\bar{i}} \nabla_{t} \psi_{+}^{j}+R_{i \bar{j} k \bar{l}} \psi_{+}^{i} \psi_{+}^{\bar{j}} \psi_{-}^{k} \psi_{-}^{\bar{l}}\right)
$$

The canonical quantization of this model gives rise to the anticommutation relations among Fermi fields at a fixed time $t$, the nontrivial part of which is

$$
\left.\left\{\psi_{-}^{i}, \psi_{-}^{\bar{i}}\right\}\right|_{t}=2=\left.\left\{\psi_{+}^{i}, \psi_{+}^{\bar{i}}\right\}\right|_{t} .
$$

Take $\psi_{+}^{i}(t), \psi_{-}^{\bar{i}}(t)$ as the creation operators and $\psi_{-}^{i}(t), \psi_{+}^{\bar{i}}(t)$ as the annihilation operators, then the Fock space that these fermion operators generate is $\wedge^{* * *}\left(T_{\Phi(t)} M \otimes \mathbf{C}\right)$. So the Hilbert space of the model (denoted by $\mathcal{H}$ ) is the space of complex differential forms, with $\psi_{-}^{i}$ acting as $\left.\mathrm{id} z^{i}\right\lrcorner, \psi_{-}^{\bar{i}}$ as i $\mathrm{d} z^{\bar{i}} \wedge$, and $\psi_{+}^{i}$ acting as $\mathrm{id} z^{i} \wedge, \psi_{+}^{\bar{i}}$ as $\left.\mathrm{id} z^{\bar{i}}\right\lrcorner$. This space admits a $\mathbf{Z} \times \mathbf{Z}$-grading, given by operators $F_{+}$and $F_{-}$, which counts the plus-fermion numbers (holomorphic degree) and the minus-fermion numbers (antiholomorphic degree) respectively.

Let $H$ be the Hamiltonian of the model, $q$ a complex number modulus one, $\bar{q}$ the complex conjugate of $q$. A standard argument involving coherent states [4] shows that

$$
\operatorname{Tr}_{\mathcal{H}}\left((-1)^{F_{-}}(-q)^{F_{+}} \mathrm{e}^{-\beta H}\right)=\int \mathrm{e}^{-S_{E}\left(\phi, \psi_{+}, \psi_{-}\right)}[\mathrm{d} \phi]\left[\mathrm{d} \psi_{-}\right]\left[\mathrm{d} \psi_{+}\right]
$$

where the path integral is over configurations where $\phi$ and $\psi_{-}$are periodic and $\psi_{+}^{i}(\beta)=$ $q \psi_{+}^{i}(0)$ and $\psi_{+}^{\bar{i}}(\beta)=\bar{q} \psi_{+}^{\bar{i}}(0)$, and

$$
S_{E}=\int_{0}^{\beta} \mathrm{d} t\left(g_{\bar{i} j} \dot{\phi}^{\bar{i}} \dot{\phi}^{j}+g_{\bar{i} j} \psi_{-}^{\bar{i}} \nabla_{t} \psi_{-}^{j}+g_{\bar{i} j} \psi_{+}^{\bar{i}} \nabla_{t} \psi_{+}^{j}-R_{i \bar{j} k \bar{l}} \psi_{+}^{i} \psi_{+}^{\bar{j}} \psi_{-}^{k} \psi_{-}^{\bar{l}}\right)
$$

is the Euclidean version of $I$ in equation (3) obtained from $-\mathrm{i} I$ by Wick-rotation:

$$
\mathrm{i} \beta \rightarrow \beta \quad \mathrm{i} t \rightarrow t .
$$

The supersymmetries of $I$ in (3) that survive in the aforementioned configurations are

$$
\begin{array}{ll}
\delta \phi^{i}=\mathrm{i} \alpha \psi_{-}^{i} & \delta \phi^{\bar{i}}=\mathrm{i} \bar{\alpha} \psi_{-}^{\bar{i}} \\
\delta \psi_{-}^{i}=-\bar{\alpha} \dot{\phi}^{i} & \delta \psi_{-}^{\bar{i}}=-\alpha \dot{\phi}^{\bar{i}} \\
\delta \psi_{+}^{i}=-\mathrm{i} \alpha \psi_{-}^{j} \Gamma_{j k}^{i} \psi_{+}^{k} & \delta \psi_{+}^{\bar{i}}=-\mathrm{i} \bar{\alpha} \psi_{-}^{\bar{j}} \Gamma_{\bar{j} \bar{k}}^{\bar{i}} \psi_{+}^{\bar{k}}
\end{array}
$$

and the corresponding supersymmetry charges are $\bar{\partial}+\bar{\partial}^{\dagger}$ (corresponding to $\alpha$ being real) and $-\mathrm{i}\left(\bar{\partial}-\bar{\partial}^{\dagger}\right)$ (corresponding to $\alpha$ being imaginary). The space of SUSY invariant states (denoted by $\mathcal{H}_{0}$ ) is identified with the space of complex harmonic forms. Then a standard argument in SUSY quantum mechanics shows that the left-hand side of (5) is independent of $\beta$ and can be simplified as

$$
\operatorname{Tr}_{\mathcal{H}_{0}}\left((-1)^{F_{-}}(-q)^{F_{+}}\right)
$$

which is obviously the left-hand side of (2) with $y=-q$. 
It remains to show that the path integral in (5) can be simplified as the right-hand side of (2), and this will be done in the following section.

\section{Evaluation of the path integral}

Since the path integral is independent of $\beta$, it is convenient to take the limit $\beta \rightarrow 0$, when many higher order interaction terms drop out of the action. As $\beta \rightarrow 0$ the penalty for time variation in the bosonic fields becomes larger, and the nonconstant boson modes are confined to narrower ranges; they are then approximated better by tangent-space variables. This allows the considerable simplification of the description of their contributions to the path integral as integrals over flat vector spaces. The two main problems in calculating the functional integral are to identify the leading terms as $\beta \rightarrow 0$ and to determine the proper integration measure.

We will first consider the integration measure for the nonzero modes. This can be determined from the free Euclidean action.

Let $q=\mathrm{e}^{\mathrm{i} \delta}$. Expanding the fields in Fourier components,

$\phi^{i}=x_{0}^{i}+\sqrt{\beta} \sum_{n \neq 0}^{\infty} a_{n}^{i} \mathrm{e}^{2 \pi n t / \beta} \quad \phi^{\bar{i}}=x_{0}^{\bar{i}}+\sqrt{\beta} \sum_{n \neq 0}^{\infty} a_{n}^{\bar{i}} \mathrm{e}^{-2 \pi n t / \beta}$

$\psi_{-}^{i}=\sqrt{\frac{\mathrm{i}}{2 \pi \beta}} \psi_{0}^{i}+\sum_{n \neq 0} \psi_{n}^{i} \mathrm{e}^{\mathrm{i} 2 \pi n t / \beta} \quad \psi_{-}^{\bar{i}}=\sqrt{\frac{\mathrm{i}}{2 \pi \beta}} \psi_{0}^{\bar{i}}+\sum_{n \neq 0} \psi_{n}^{\bar{i}} \mathrm{e}^{-\mathrm{i} 2 \pi n t / \beta}$

$\psi_{+}^{i}=\mathrm{e}^{\mathrm{i}(\delta / 4+\delta t / \beta)} \eta_{0}^{i}+\sum_{n \neq 0} \eta_{n}^{i} \mathrm{e}^{\mathrm{i}(2 \pi n+\delta) t / \beta} \quad \psi_{+}^{\bar{i}}=\mathrm{e}^{\mathrm{i}(\delta / 4-\delta t / \beta)} \eta_{0}^{\bar{i}}+\sum_{n \neq 0} \eta_{n}^{\bar{i}} \mathrm{e}^{-\mathrm{i}(2 \pi n+\delta) t / \beta}$.

The factors of $\sqrt{\beta}$ and $\sqrt{\frac{\mathrm{i}}{2 \pi \beta}}$ in the mode expansions have the effect of removing all $\beta$ dependence from the integration measure and setting the zero-mode measure to 1 .

We will integrate first all nonzero modes of all fields, then all fermionic zero modes, all of which are tangent-space variables, and finally we integrate over $x_{0}^{\bar{i}}$ and $x_{0}^{i}$. Since the path integral is invariant under changes in coordinates, we may perform the tangent-space integrations using normal coordinates centred at the corresponding point in $M$. This means that we may set $x_{0}^{i}=0$ when performing these integrations.

Choosing a geodesic normal coordinate system around point $x_{0}$, the action reads

$$
S_{E}=S_{0}+S_{1}+O(\beta)
$$

where

$$
S_{0}=\sum_{n}\left[(2 \pi n)^{2} a_{n}^{\bar{i}} a_{n}^{i}+\mathrm{i} 2 \pi n\left(\psi_{n}^{\bar{i}} \psi_{n}^{i}+\eta_{n}^{\bar{i}} \eta_{n}^{i}\right)\right]
$$

and

$S_{1}=\sum_{n \neq 0}\left[\mathrm{i} \Omega_{\bar{k} l}\left(\mathrm{i} n a_{n}^{\bar{k}} a_{n}^{l}\right)-\frac{\mathrm{i} \Omega_{\bar{k}} l}{2 \pi} \eta_{n}^{\bar{k}} \eta_{n}^{l}+\mathrm{i} \delta \eta_{n}^{\bar{i}} \eta_{n}^{i}\right]+\mathrm{e}^{\mathrm{i} \delta / 2}\left(-\frac{\mathrm{i} \Omega_{\bar{k}} l}{2 \pi} \eta_{0}^{\bar{k}} \eta_{0}^{l}+\mathrm{i} \delta \eta_{0}^{\bar{i}} \eta_{0}^{i}\right)$

with $\Omega_{\bar{k} l}=R_{\bar{i} j \bar{k} l} \psi_{0}^{\bar{i}} \psi_{0}^{j}$.

The integration measure is

$$
[\mathrm{d} x]\left[\mathrm{d} \psi_{-}\right]\left[\mathrm{d} \psi_{+}\right]=\prod_{i} \mathrm{~d} x_{0}^{i} \mathrm{~d} x_{0}^{\bar{i}} \mathrm{~d} \psi_{0}^{i} \mathrm{~d} \psi_{0}^{\bar{i}} \mathrm{~d} \eta_{0}^{i} \mathrm{~d} \eta_{0}^{\bar{i}} \mathrm{~d} \mu^{i}
$$

where

$$
\mathrm{d} \mu^{i}=\prod_{n \neq 0}\left(\frac{1}{2 \pi \mathrm{i}} \mathrm{d} a_{n}^{i} \mathrm{~d} a_{n}^{\bar{i}} \mathrm{~d} \psi_{n}^{i} \mathrm{~d} \psi_{n}^{\bar{i}} \mathrm{~d} \eta_{n}^{i} \mathrm{~d} \eta_{n}^{\bar{i}}\right)
$$


and is determined by requiring

$$
\int \prod_{i} \mathrm{~d} \mu^{i} \mathrm{e}^{-S_{0}}=1
$$

Let $\Omega=\left[\Omega_{\bar{k} l}\right]_{1 \leqslant k, l \leqslant d}$ be the anti-Hermitian curvature matrix, $I$ the identity matrix, integrating $\mathrm{e}^{-S_{E}}$ over $\prod_{i} \mathrm{~d} \eta_{0}^{i} \mathrm{~d} \eta_{0}^{\bar{i}} \mathrm{~d} \mu^{i}$, we obtain (in the limit $\beta \rightarrow 0$ )

$$
\operatorname{det}\left(\mathrm{e}^{\mathrm{i} \delta / 2}\left(\frac{\mathrm{i} \Omega}{2 \pi}-\mathrm{i} \delta I\right)\right) \prod_{n>0} \operatorname{det}^{-1}\left(I+\frac{\left(\frac{\mathrm{i} \Omega}{4 \pi}\right)^{2}}{(n \pi)^{2}}\right) \operatorname{det}\left(I+\frac{\left(\frac{\mathrm{i} \Omega}{4 \pi}-\mathrm{i} \frac{\delta}{2} I\right)^{2}}{(n \pi)^{2}}\right) .
$$

To evaluate the above expression, we assume $\Omega$ can be put in the diagonal form,

$$
\Omega=\left(\begin{array}{ccc}
-\mathrm{i} \Omega_{1} & & \\
& \ddots & \\
& & -\mathrm{i} \Omega_{d}
\end{array}\right)
$$

Then we obtain (using formula $\sinh x=x \prod_{n>0}\left(1+\left(\frac{x}{n \pi}\right)^{2}\right)$ )

$$
\prod_{i} \frac{\Omega_{i} / 2 \pi}{\sinh \left(\Omega_{i} / 4 \pi\right)} \sinh _{y}\left(\Omega_{i} / 4 \pi\right)
$$

where $y=-\mathrm{e}^{\mathrm{i} \delta}$ and $\sinh _{y}(x)=\frac{\mathrm{e}^{x}+y \mathrm{e}^{-x}}{2}$. This has a power series expansion which terminates because of the Fermi statistics of the zero modes of $\psi_{-}$. Integrating it over $\prod_{i} \mathrm{~d} \psi_{0}^{i} \mathrm{~d} \psi_{0}^{\bar{i}}$ projects out the coefficient of $\prod_{i} \psi_{0}^{i} \psi_{0}^{\bar{i}}$ in the integrand, i.e. the top form, which is proportional to the volume form on $M$. For the form (15) of $\Omega$, the path integral reduces to

$$
\left.\int_{M} \prod_{i} \frac{\Omega_{i} / 2 \pi}{\sinh \left(\Omega_{i} / 4 \pi\right)} \sinh _{y}\left(\Omega_{i} / 4 \pi\right)\right|_{\text {top form }} .
$$

Considering more general forms for $\Omega$, we note that expression (14) can be seen as a sum of traces of products of curvature forms, i.e. a polynomial in Chern classes. The splitting principle then states that to calculate this polynomial, we may specialize to the case evaluated explicitly above, where the vector bundle in question splits into a sum of line bundles.

\section{References}

[1] Atiyah M F and Singer I M 1963 Bull. Am. Math. Soc. 69422 Atiyah M F and Singer I M 1968 Ann. Math. 87484 Atiyah M F and Singer I M 1968 Ann. Math. 87546

[2] Alvarez-Gaume L 1983 Supersymmetry and the Atiyah-Singer index theorem Commun. Math. Phys. 90161

[3] Friedan D and Windey P 1984 Supersymmetric derivation of the Atiyah-Singer index theorem and the chiral anomaly Nucl. Phys. B 235395

[4] Sakita B 1985 Quantum Theory of Many-Variable Systems and Fields (Singapore: World Scientific)

[5] Witten E 1992 Mirror manifolds and topological field theory Essays on Mirror Manifolds (Hong Kong: International) pp 120-58 